\title{
On what is bound in figures and grounds
}

\author{
Mary A. Peterson and Jee Hyun Kim \\ University of Arizona, Tucson, USA
}

\begin{abstract}
All else being equal, regions providing a quick, good match to object memories are likely to be seen as shaped figures rather than as shapeless grounds. Good matches to object memories occur only if the parts are properly bound spatially. If object memories are accessed in the course of perceptual organization even for regions ultimately seen as shapeless grounds, then binding of parts cannot require attention to figures. Novel silhouette primes were shown immediately before real or novel line drawings. The silhouette contours of half of the primes preceding real line drawings sketched a known shape on the outside, which nevertheless appeared to be a shapeless ground. At short prime-line drawing SOAs, observers took longer to categorize real line drawings following these experimental primes than control primes, suggesting that parts are properly bound spatially for regions perceived to be shapeless grounds, and further that object memories matching grounds are inhibited.
\end{abstract}

Early in the course of perceptual processing, certain regions in the visual field are assigned figural status and others are assigned ground status (see Figure 1). Figures and grounds have different properties. Figures have a definite shape that, if familiar, can be recognized; they appear to be things or objects. Grounds are not shaped by any contours they share with figures; they appear to simply continue behind the figures near those contours. Kahneman and Treisman (1984) proposed that when an object is first sensed (presumably after figure assignment), an "object file" is opened as a precursor to a perceived object. Information about object features (e.g., motion, brightness, colour, size) and object identity is entered into the object file as it becomes available. Object files segregate information belonging to different objects (Kahneman \& Treisman, 1984); attention then binds together various features of objects (such as colour and form) (Treisman \& Gelade, 1980).

Please address all correspondenc e to Dr. M.A. Peterson, Department of Psychology, University of Arizona, Tucson, AZ 85721, USA. Email: mapeters@u.arizona.ed u

This research was supported by a National Science Foundation grant (BCS 9906063) to the first author. We thank Melissa Lin, Logan Trujillo, and especially Daniel Lampignano for their help with these experiments. Tia Kalla did an excellent job drawing the real line drawings we used to supplement the Snodgrass and Vanderwart set. 


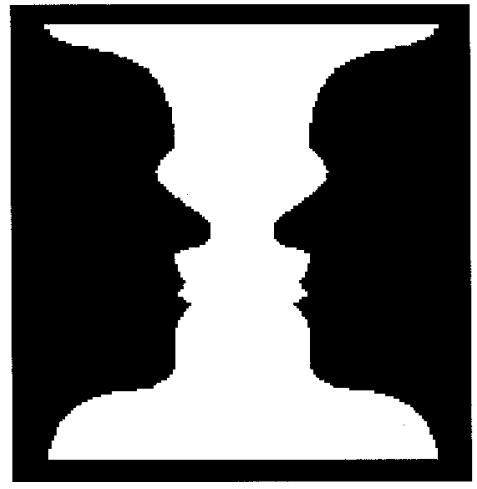

Figure 1. A. The Rubin vase-faces display illustrates the coupling between figural status and conscious recognition. As one steadily observes this display, figural status is alternately assigned to the black and white regions. The face profiles can be seen and recognized as such only when the black regions appear to be figure. When the white region appears to be the figure, the black region appears shapeless near the contours it shares with the black region. Similarly, the vase (goblet) can be seen/recognized only when the white region appears to be the figure; it cannot be seen/recognized when the black regions appear to be figures at the borders they share with the white region.

Wolfe and Bennett (1997) interpreted their visual search results as evidence that the parts within an object file remain spatially unordered unless attention is allocated to the location of the object file. Hence, an object in an unattended object file would be indistinguishable from a scrambled version of the same object. On this account, attention serves to "bind" the parts of the figure in the object file into their proper spatial relationships (Wolfe \& Bennett, 1997). It is well known that good access to object memories requires the parts of the object to be arranged properly in space: Scrambled versions of objects, in which the parts have been spatially rearranged, cannot be correctly identified (Biederman, 1987; Cave \& Kosslyn, 1993; Hummel \& Biederman, 1992; Peterson, Harvey, \& Weidenbacher, 1991). Thus, on Wolfe and Bennett's account, once attention binds together the parts of a figure within an object file, the condition s have been established for successful access to object memories.

Implicit in the reasoning about object files are the assumptions that object files (1) are established for figures but not for grounds, and (2) are precursors for access to object memories. From this line of reasoning, the assumption that object memories are accessed for figures and not for grounds follows. This assumption is common among perception researchers; it arises in part from the fact that grounds appear shapeless near the contours they share with figures (see also Peterson, 1999). However, the fact that grounds appear shapeless does not answer to the question of whether or not grounds were matched to object memories in the course of perceptual organization. Grounds might appear shapeless because of inhibitory connections between processes operating on opposite sides of a contour (Peterson, de Gelder, Rapcsak, Gerhardstein, \& 
Bachoud-Lévi, 2000). In the two experiments presented here, we investigated whether or not object memories are accessed in the course of perceptual organization for regions ultimately perceived to be shapeless grounds. Evidence indicating that access to object memories has occurred will be taken to imply that the parts of grounds are bound together properly prior to the assignment of figure and ground status.

In the present experiments, figure-ground displays were presented as primes for line drawing targets. The figure-ground primes were small, symmetric, enclosed, black silhouettes shown centred on a much larger white screen. (Samples are shown in Figure 2.) Participants were asked to just look at the primes; they made no response to them. Their task was to quickly judge whether a line drawing shown immediately after the prime portrayed a real or a novel object. (Sample line drawings are shown in Figure 3.) The critical trials were those involving line drawings of real objects. Half of the line drawings of real objects were preceded by control primes and half were preceded by experimental primes.

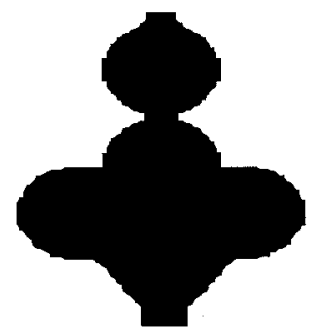

A.

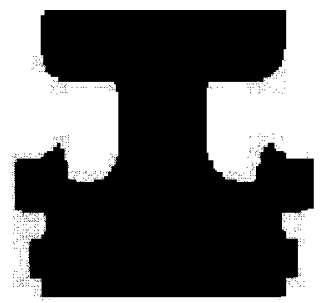

c.

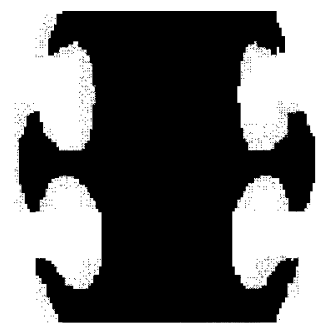

B.

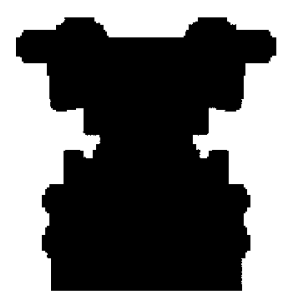

D.

Figure 2. Samples of the silhouettes shown before the line drawings. These "control" silhouettes are low in denotivity along both side of their contours in that neither side of the contour provides a good match to an object memory. 


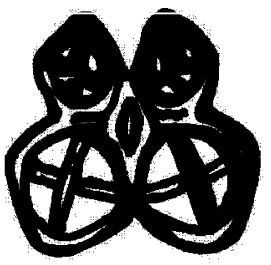

A.

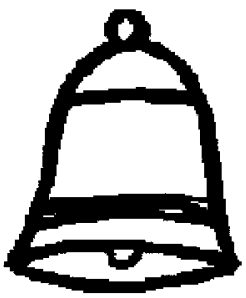

C.
B.

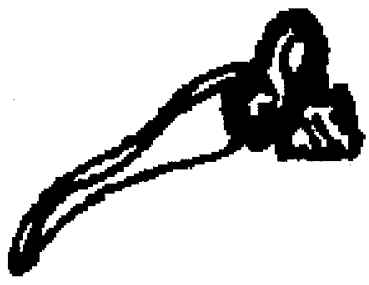

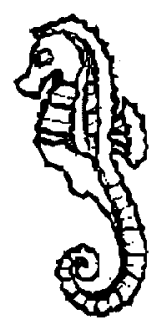

D.

Figure 3. Line drawings of novel objects (A and B) and real objects (a bell in C and a sea horse in D) used in the experiment.

The contours of control primes sketched novel objects along both the black figure side (the inside) and the white ground side (the outside) (see Figure 3). The contours of experimental primes also sketched novel objects on the figure side (the inside). The experimental primes differed from the control primes in that their contours sketched a portion of a known object along the ground side (the outside) of the silhouette. (Samples are shown in Figure 4.) The portions of known objects sketched on the ground side of the contours of experimental silhouettes were sufficient to support correct identification by pilot observers who saw these regions as figure in other displays. However, the observers in the current experiments saw the white surrounds as shapeless grounds to the black silhouette figures (see later).

The line drawings shown after the experimental primes portrayed an object from the same basic level category as the object sketched on the ground side of the contour of the silhouette prime. ${ }^{1}$ If, in the course of perceptual organization,

${ }^{1}$ The contours of the experimental silhouettes were not used to generate the line drawings. This is because we were interested in assessing whether or not pre-existing object memories were accessed during figure assignment. We were not interested in testing the different question of whether or not memories are establishe d for novel ground regions (see Treisman \& DeSchepper, 1996, and General Discussion, later). 


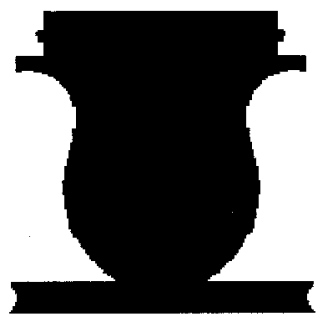

A.

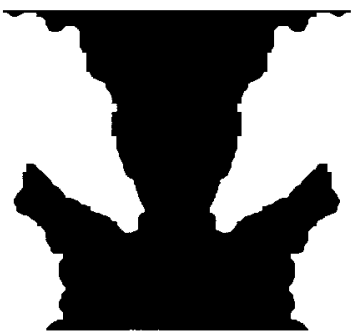

C.

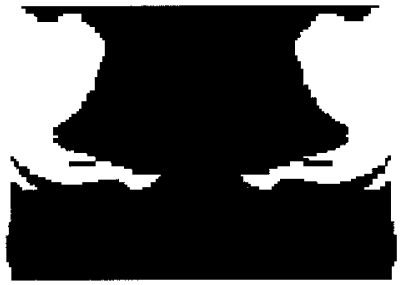

B.

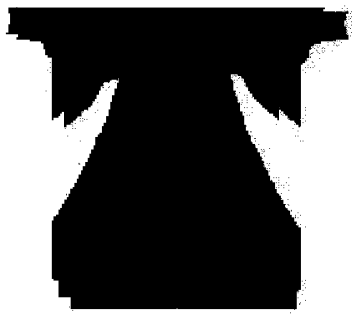

D.

Figure 4. Sample experimental primes. The contours of the silhouettes sketch parts of the outlines of bells (A), elephants (B), sea horses (C), and coffee pots (D) along the outer, ground, side. Hence, the contours of these silhouettes were high in denotivity along the outside in that they provided a good match to an object memory.

object memories are accessed for regions subsequently perceived to be shapeless grounds, then reaction times to correctly classify the line drawings as real objects should differ depending upon whether they appear after an experimental or a control prime. A specific prediction about the direction of the difference arises from a model proposed by Peterson et al. (2000), which is one of a class of models predicting cross-contour inhibition in figure assignment (see also Sejnowski \& Hinton, 1987; Vecera \& O' Reilly, 1998). The Peterson et al. model predicts that object memories accessed for one side of a contour will be inhibited when more or stronger cues favour the interpretation that the other 
side is a shaped figure. The magnitude of the hypothesized inhibition depends on the relative strength of the cues favouring the interpretation that the figure lies on the other side of the contour. The hypothesized inhibition may be reflected in response latency. If it is, then participants will take longer to accurately report that line drawings portray familiar objects when they are preceded by experimental primes rather than by control primes. The proposed inhibition is expected to occur early in processing when figure assignment is determined, and to be short-lived. We tested this hypothesis by separating the primes and the line drawing targets by SOAs of different durations.

It is important to note that, although the real objects sketched along the outside of the vertical contours of the experimental primes may be clearly visible to the readers of this paper, they were not visible to the observers in the experiment. ${ }^{2}$ A relatively large number of cues favoured the interpretation that the black silhouette, rather than the surrounding white region, was the shaped entity in the visual field (i.e., the figure). Silhouettes were always reflectionally symmetric around a vertical axis, and were much smaller in area than, and enclosed by, the white surround. Regions with these Gestalt configural properties are more likely to be seen as figure than adjacent regions lacking these properties (for a review see Peterson, 2000). In addition, participants were instructed to attend to the silhouettes, which appeared where they were fixating their eyes at the beginning of a trial. Fixated and attended regions are likely to be seen as figures (Baylis, Tipper, \& Houghton, 1997; Driver \& Baylis, 1998; Hochberg, 1971; Peterson \& Gibson, 1994a; Rubin, 1915/1958). Finally, observers expected to see a silhouette of a novel shape on every trial before the line drawings. Hence, the setting and the participants' expectations contributed to the likelihood that the black silhouette would be seen as a figure. Thus, for the silhouette primes, a large number of cues favoured the interpretation that the figure lay on the black, inner side of the contour, whereas only one cue, the object memory cue, favoured the interpretation that the figure lay on the white, outer side of the contour. It has been shown that object memory cues do not necessarily the dominate figure assignment when a single competing cue is present (Peterson, 1994b, 2000; Peterson et al., 2000; Peterson \& Gibson, 1993, 1994b). With multiple competing cues present, as in these displays, most observers perceived the black silhouette primes as the shaped entities in the visual field and the adjacent white regions as shapeless grounds. These conditions allowed us to test whether or not object memories are accessed for regions ultimately perceived to be shapeless grounds.

${ }^{2}$ Some observers did see the shapes in the ground. The data obtained from those participants were excluded before they were analysed. (See Methods section for details.) 


\section{EXPERIMENT 1}

\section{Method}

Participants. Participants were 34 undergraduate students who enrolled in the experiment to partially fulfill a requirement for their introductory psychology class. Before the data were analysed, twelve participants were excluded: One because a system error occurred during the experiment, one because he was uncooperative, and nine because they reported becoming aware of the shapes in the grounds of the priming stimuli, typically after participating in more than two blocks of trials. ${ }^{3}$ Data acquired from one other participant were excluded because he failed to make a response to $33 \%$ of the novel stimuli in one block of trials.

Stimuli and apparatus. The line drawings $(N=84)$ depicted 42 real and 42 novel objects. Novel objects were drawn from Kroll and Potter (1984). Many of the line drawings of real objects were taken from the Snodgrass and Vanderwart (1989) set; others were created for this experiment. Line drawings varied in height from $1.2^{\circ}$ to $4.5^{\circ}$, and in width from $1.0^{\circ}$ to $7.8^{\circ}$. Line drawings of real objects were divided into two sets (A and B). (See the Appendix for a listing of the objects in sets A and B.) For half of the participants, A was the primed set and $\mathrm{B}$ was the control set (i.e., A line drawings were preceded by experimental silhouettes and B line drawings were preceded by control silhouettes). For the other half of the subjects, this assignment was reversed. Across sets $\mathrm{A}$ and $\mathrm{B}$, line drawings were matched for area, axis of elongation, word frequency of the object's name, number of symmetric versus asymmetric drawings, and number of living versus non-living objects. An additional 11 line drawings were used on practice trials.

The silhouettes were as described earlier; 42 were experimental silhouettes. These experimental silhouettes portrayed a portion of one of the known objects in either set A or set B along their outside edge. The experimental silhouettes were divided into two sets, $\mathrm{A}$ and $\mathrm{B}$, matching the line drawing sets $\mathrm{A}$ and $\mathrm{B}$. A given participant saw only 21 of the experimental silhouettes (either set A or set B silhouettes). The remaining 63 silhouettes shown on experimental trials ( $N=$ 84 ) were control silhouettes. Of these, 20 were inverted versions of other control stimuli in the set. Each silhouette subtended a visual angle of $3.4^{\circ}$ in height; they ranged in width from $1.0^{\circ}$ to $4.1^{\circ}$. Silhouettes were shown centred on a white screen, $23.3^{\circ}$ wide by $16.4^{\circ}$ high.

${ }^{3}$ Because a within-subjects design was used in this experiment, observers saw the stimuli repeatedly across different conditions. Observers who saw the shapes in the white surround region of the primes typically did so after being exposed to the stimuli numerou s times. It is likely that, for these observers, the factors of attention and expectancy ceased to operate in favour of seeing the black silhouette as figures as the experiment progressed. 
Stimuli were viewed from a distance of approximately $98 \mathrm{~cm}$ on an Optiquest monitor (Model V773). The experiment was run on an ACT personal computer equipped with a $166 \mathrm{MHz}$ processor. Responses were made on a custom response box. The presentation software was the DMDX software developed by Jonathan C. Forster at the University of Arizona.

Procedure. During the experiment, a chin rest was used to keep participants' heads at a constant distance from the screen. Participants were told that their task was to distinguish between line drawings of real and novel objects as quickly and as accurately as possible. (Examples of each were shown.) They were told that a black silhouette would precede each line drawing and they would see many different black silhouettes, all of which would be novel and symmetrical around a vertical axis. Participants were asked to look at the silhouette, but to make no response to it. Each trial began with the presentation of a small fixation cross in the centre of the screen, on which participants were asked to fixate. When they were fixating properly, and were ready for the trial to begin, participants pressed a foot pedal. A black silhouette then appeared centred at fixation for $50 \mathrm{~ms}$, and was followed by a blank white screen that varied in duration for each of five blocked SOA conditions (SOAs = 83, 200, 350, 500 , and $650 \mathrm{~ms}$ ). Next, a line drawing appeared (also centred at fixation) and remained on the screen until a response was made (or until $1.5 \mathrm{~s}$ had elapsed). Participants indicated whether the line drawing portrayed a real or novel object by pressing one of two keys. Pressing a key with the dominant hand indicated a real object; pressing a key with the non-dominant hand indicated a novel object. Reaction times (RTs) were measured for correct responses from the onset of the line drawing target. Following the participant's response, regardless of accuracy, the fixation cross for the next trial appeared. ${ }^{4}$ No feedback was given.

We used a $50 \mathrm{~ms}$ exposure duration for the prime because pilot testing indicated that duration was sufficient for observers to obtain a clear percept of the black silhouette as figure. A $33 \mathrm{~ms}$ blank screen was inserted following the prime display in the shortest SOA condition so that all conditions included a blank between the prime and the target displays. This resulted in a smaller difference between the first two SOA conditions $(117 \mathrm{~ms})$ than between the other SOA condition $(150 \mathrm{~ms})$. The $83 \mathrm{~ms}$ condition allows us to probe for evidence of access to object memories close to when the figure assignment was made for the prime displays. The longer SOA conditions allowed us to explore the time course of any priming we obtained.

All participants were exposed to all five SOA conditions. The order of SOA conditions was counterbalanced across subjects via a Latin square. Each SOA condition was comprised of two blocks, a priming block followed by an

${ }^{4}$ If no response was made within $1.5 \mathrm{~s}$, the line drawing was removed and replaced by the fixation cross for the next trial. 
uncoupling block. Within each block there were 11 practice trials followed by 84 experimental trials (comprised of 42 real and 42 novel line drawings). Neither silhouette nor line drawing stimuli used on practice trials, were included in the experimental trials, with the exception of two silhouettes that were shown in an inverted orientation on experimental trials. In the experimental block, an experimental prime silhouette preceded 21 of the real line drawings; a control silhouette preceded the other 21. Control silhouettes preceded all 42 novel line drawings. Trial types were randomly presented. No silhouettes or line drawings were repeated in the priming block. All silhouettes and line drawings were seen a second time in the uncoupling block. ${ }^{5}$ During the uncoupling blocks, experimental primes preceded novel line drawings and control primes preceded all real object line drawings. (All silhouettes and line drawings were re-paired for the uncoupling block to prevent observers from learning paired associations between silhouettes and line drawings over the course of the experiment.) The results obtained on these uncoupling blocks will not be reported because the use of randomly presented trials prevented us from controlling the duration between the presentation of a silhouette prime and its paired line drawing during the uncoupling block. Observers were given a short break between the experimental and uncoupling blocks for a given SOA condition and a 5-min break between SOA blocks. Participation in this experiment took approximately 1.5 hours.

After they had participated in all five pairs of priming and uncoupling blocks, participants were asked whether they had seen any known objects in the white surrounds. As they were questioned, participants were shown pictures of three silhouettes and, for each, the object portrayed along the outer contour in the white surround was pointed out to them. (One of these silhouettes was designed especially for the debriefing so that the known object portrayed in the surround could be seen easily.) During these post-experiment questions, many observers formed the impression that they were supposed to see the objects portrayed on the outside of the silhouettes, and were embarrassed to admit they had not seen them. We certainly did not intend that observers see the white regions as shaped during the experiment. However, the fact that many participants felt they should have seen the shapes in the white surrounds when we questioned them gave us some confidence that those who said they had not seen shapes in the white regions were reporting honestly. At this stage, nine of the observers reported that they did see the shape portrayed by the white region adjacent to the black silhouette; they typically reported seeing shapes in the white regions after participating in more than two SOA condition s (hence, after seeing the silhouettes four times). The data from these participants were not analysed since we are interested in investigating whether or not priming can be obtained from

${ }^{5}$ One subject was not run in the $500 \mathrm{~ms}$ condition of the uncoupling block due to experimenter error. 
regions perceived to be shapeless grounds, and these observers had potentially seen the white regions as shaped figures. ${ }^{6}$

\section{Results}

Mean RTs were calculated for each participant in each condition (primed and unprimed real objects, and novel objects). RTs that were more than two standard deviations from the individual's condition mean were excluded. This process was repeated until no outliers remained. Mean RTs obtained for both primed (P) and unprimed (UP) real object responses are shown in Table 1. A repeated measures analysis of variance (ANOVA) on these means showed a main effect of condition, $F(2,21)=35.09, p<.001$, indicating that RTs were longer for accurate novel responses $(584 \mathrm{~ms})$ than for accurate real responses (515 ms).

For each participant, the index of priming was the difference between the mean reaction time recorded on Unprimed and Primed trials (UP-P). These difference scores were entered into a one-way ANOVA with 5 SOA levels. A main effect of SOA was obtained, $F(4,21)=2.474, p=.051$. We conducted planned $t$-tests to investigate whether or not significant priming was obtained in each of the SOA conditions. As expected, observers' latency to respond to primed line drawings was longer than their latency to respond to unprimed line drawings in the shortest ( $83 \mathrm{~ms}$ ) SOA condition, $\mathrm{UP}-\mathrm{P}=-18.74 \mathrm{~ms}, t(21)$ $=-1.90, p<.05$, one tailed. This finding indicates that object memories matching the white grounds were accessed in the course of perceptual organization. Furthermore, the fact that the difference score is negative indicates that object memories matching regions seen as shapeless grounds are inhibited, as predicted by Peterson et al. (2000). No differences between the latency to respond to primed versus unprimed line drawings was observed for the 200,350, or 650 $\mathrm{ms}$ SOA, all $p \mathrm{~s}>.20$. Post hoc tests indicated that the (UP $-\mathrm{P})$ differences obtained in the $83 \mathrm{~ms}$ condition and the $350 \mathrm{~ms}$ condition were significantly different, $p<.03$, suggesting that no measurable inhibition remained in the $350 \mathrm{~ms}$ condition. Thus, the inhibition appears to be short-lived, as predicted by Peterson et al. (2000).

Unexpectedly, in the $500 \mathrm{~ms}$ SOA condition, observers' latency to respond to primed line drawings was again longer than their latency to respond to

\footnotetext{
${ }^{6}$ Because of the obviou s demand character of the post-experimen $t$ questions, it is not clear that all of these observers really saw the shape sketched in the white surrounds. They often reported seeing shapes that were not sketched in the surround s of the experimental silhouettes (i.e., shapes in the control set). Nevertheless, we did not inspect the data from any of the observers who reported seeing shapes in the white surround.
} 


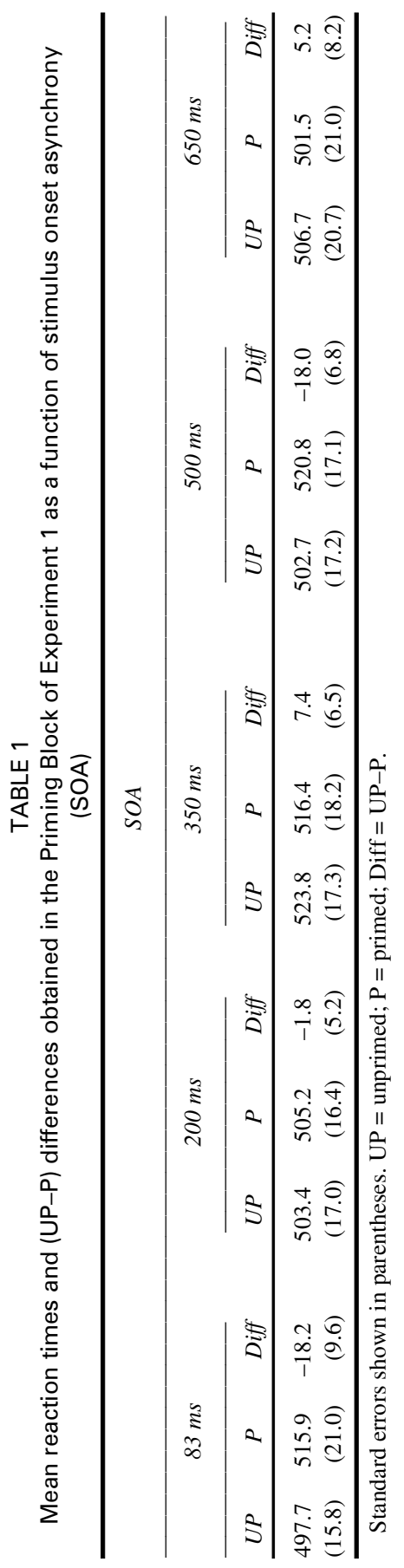


unprimed line drawings, $\mathrm{UP}-\mathrm{P}=-18.0, t(20)^{7}=2.67, p<.02$, two tailed. The reappearance of inhibition in the $500 \mathrm{~ms}$ condition is surprising. We note that inhibition in the $500 \mathrm{~ms}$ condition was most evident among observers who saw the $500 \mathrm{~ms}$ condition either third or fourth in the sequence of conditions, and, hence, may be due to the repetition of the stimuli across three or four blocks rather than to the SOA itself. Unfortunately, the number of participants who received each order was too small to permit a sensitive test of this hypothesis. However, in Experiment 2, we used a between-subjects design in order to eliminate order effects, which may have contaminated the data obtained in other SOA conditions as well.

\section{EXPERIMENT 2}

\section{Method}

Participants. Participants were 132 undergraduate students who enrolled in the experiment to partially fulfill a requirement for their introductory psychology class. Before the data were analysed, 19 participants were excluded: One because of a shoulder injury that might have interfered with her ability to respond, one because he had been told about the experimental design before arriving at the laboratory, and 17 because they reported becoming aware of the shapes in the grounds of the priming stimuli. The percentage of students dropped for the last reason was half as large in Experiment $2(13 \%)$ as in Experiment $1(26 \%)$, probably because of the use of a between-subjects design in Experiment 2. Fifteen of the participants who saw the shapes in the surround were exposed to the primes for $350 \mathrm{~ms}$ or more, ample time to make an eye movement, and, hence, to change the balance of cues favouring the black and white regions as figures. (The other two subjects were in the $200 \mathrm{~ms}$ condition.)

Stimuli and apparatus. The stimuli and apparatus used in Experiment 2 were much the same as those used in Experiment 1, although new control silhouettes were added so that the experimental primes were not repeated in the uncoupling block, and none of the silhouettes was used in both an upright and an inverted orientation in the priming block. There were 84 control silhouettes, and 42 experimental silhouettes. As in Experiment 1, a given subject saw only 21 of the experimental silhouettes. Of the 84 control silhouettes, 63 were shown in both the priming block and the uncoupling block (they were paired with different line drawings in the uncoupling block); 21 were shown in the uncoupling block only as replacements for the 21 experimental silhouettes. In Experiment

\footnotetext{
${ }^{7}$ One subject was dropped from the analysis of the $500 \mathrm{~ms}$ condition of the priming block because of a high error rate. For the ANOVA, the condition mean was entered into the cell so there would be no missing data.
} 
2, each silhouette was matched as closely as possible to the corresponding line drawing in curvature and size, while preserving the resolution and aspect ratio. The silhouettes subtended $2.1^{\circ}-5.4^{\circ}$ in height and $1.6^{\circ}-5.6^{\circ}$ in width. The line drawings subtended $2.1^{\circ}-3.1^{\circ}$ in height and $0.8^{\circ}-4.3^{\circ}$ in width. Across priming sets $\mathrm{A}$ and $\mathrm{B}$, line drawings were matched for curvature (which had not been done in Experiment 1). Thus, the sets used in Experiment 2 were somewhat different from those used in Experiment 1 (leaf and pine tree were switched into set A and lamp and palm tree were switched into Set B). In the course of matching the two sets for curvature, they became slightly unmatched in the number of living things.

Procedure. When participants entered the laboratory they were assigned to one of the SOA conditions using a pseudo-random procedure that balanced experimenter and time of day across SOA conditions. In this experiment, the primes were exposed for the full duration of the SOA. Under these conditions, the shortest SOA used was $50 \mathrm{~ms}$, and all adjacent SOA conditions differed by $150 \mathrm{~ms}$. There were 23 participants in each of the $50 \mathrm{~ms}, 350 \mathrm{~ms}$, and the $650 \mathrm{~ms}$ conditions and 21 participants in each of the $200 \mathrm{~ms}$ and $500 \mathrm{~ms}$ conditions.

Each observer participated in two blocks of trials, a priming block followed by an uncoupling block. Within each block, trials were presented in a random order. The instructions for this experiment were presented on the computer screen. Participants were able to ask the experimenter questions regarding the experiment at any time while reading the instructions or while participating in the practice trials. They were then left alone to perform the experimental trials. Participation in this experiment took approximately $20 \mathrm{~min}$.

\section{Results}

Mean RTs were calculated for each participant in each condition (primed and unprimed real objects, and novel objects). RTs more than two standard deviations from the individual's condition mean were excluded. This process was repeated until no outliers remained. Mean RTs obtained for both primed and unprimed real object responses are shown in Table 2. For each participant, the difference was taken between the mean RT recorded on primed and unprimed trials.

It was obvious upon inspection of the overall means and standard errors that there were no differences between primed (P) and unprimed (UP) RTs in any of the SOA conditions. We noted, however, that much longer RTs were obtained in Experiment 2 than in Experiment 1. Consider the $83 \mathrm{~ms} \mathrm{SOA}$ condition where inhibition was observed in Experiment 1. The mean UP RT was $516 \mathrm{~ms}$, whereas the mean UP RT in the comparable short SOA condition of Experiment 2 (i.e., the $50 \mathrm{~ms}$ condition) was $573 \mathrm{~ms}$, a difference of almost $60 \mathrm{~ms}$. Inhibition applied to object memories accessed for regions determined to be 


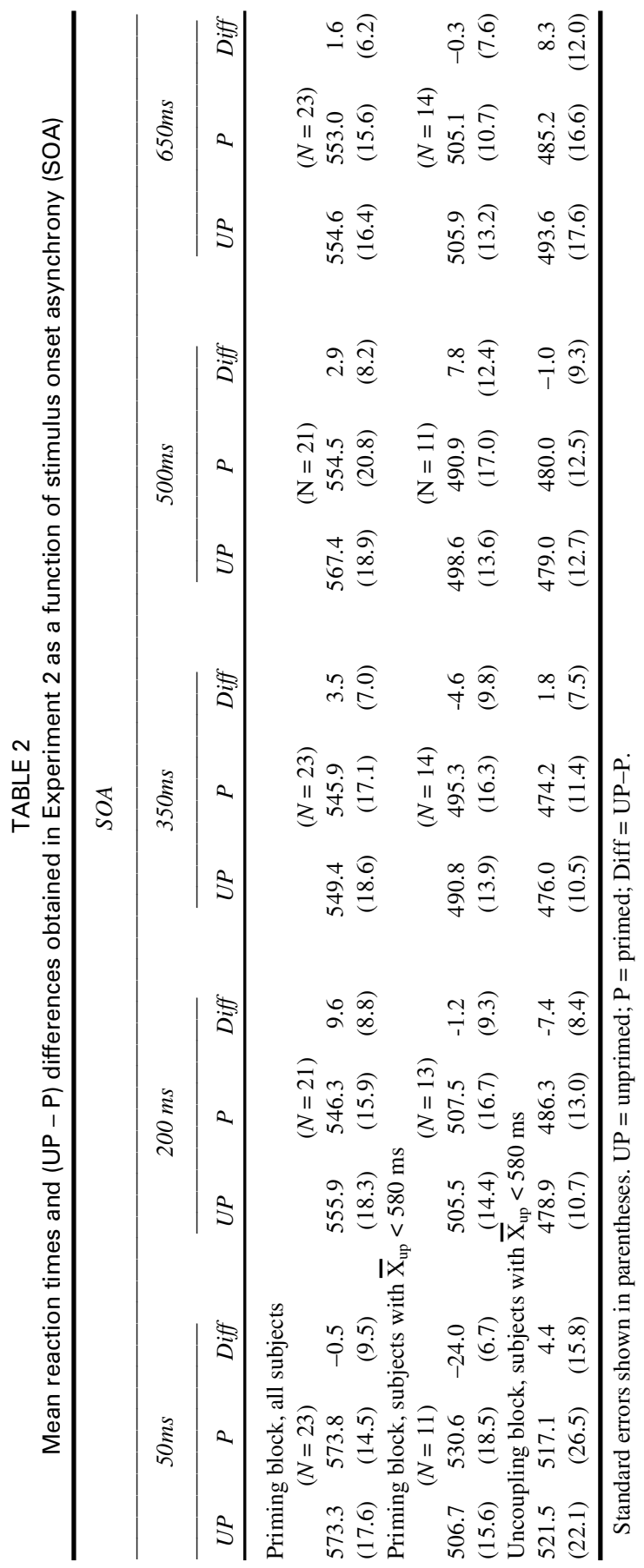


grounds is expected to be short-lived. Therefore, it would be difficult to observe inhibition if RTs are too long. Indeed, in both the $50 \mathrm{~ms}$ and $200 \mathrm{~ms}$ condition s of Experiment 2, the magnitude of the difference score (UP - P) was correlated with the mean RT on UP trials, $r \mathrm{~s}=0.57$ and 0.50 in the $50 \mathrm{~ms}$ and the $200 \mathrm{~ms}$ conditions, respectively, $p s<.02$. In other words, the longer the observers took to respond to the line drawings shown on unprimed trials, the more positive the (UP - P) difference score was (i.e., the less negative it was).

In Experiment 1, 77\% of the observers had mean UP RTs that were less than $630 \mathrm{~ms}$ from the onset of the silhouette, whereas only $48 \%$ of the observers in Experiment 2 had mean UP RTs below that limit. Accordingly, we separately examined the difference scores of those observers in Experiment 2 whose UP RTs were less than $630 \mathrm{~ms}$ from the onset of the silhouette (i.e., observers with UP RT means less than or equal to $580 \mathrm{~ms}$ in the $50 \mathrm{~ms}$ condition). The mean RTs for this subset of participants is shown in the middle panel of Table 2. Planned comparisons showed that in the $50 \mathrm{~ms}$ condition, the subset of observers with mean UP RTs $<580 \mathrm{~ms}$ took significantly longer to respond to line drawings on primed trials than on unprimed trials, $t(10)=-3.567, p<.01$, two tailed. Thus, Experiment 2 replicates Experiment 1 when responses within the same RT range are examined. ${ }^{8}$ Like Experiment 1, Experiment 2 indicates that object memories matching regions ultimately perceived to be shapeless grounds are inhibited.

Planned comparisons were conducted on the subset of observers whose mean UPRTs were $\leq 580 \mathrm{~ms}$ in the other SOA conditions as well. No significant (UP - P) difference scores were obtained in any other SOA condition, all $t \mathrm{~s}<$ 1.0. Thus, inhibition does not seem to be sufficiently long-lived to be observed using this paradigm when the silhouettes are exposed for $200 \mathrm{~ms}$ or longer.

Performance on the uncoupling block provides a different, within-subjects measure of the longevity of priming. ${ }^{9}$ Suppose residual priming lasts the approximately 2-3 min between the presentation of a primed line drawing in the priming block and the presentation of the same line drawing in an unprimed condition in the uncoupling block. Then, RTs for the previously primed line drawings should be longer than the previously unprimed line drawings. No such difference was found in the $50 \mathrm{~ms}$ condition, where significant inhibition

\footnotetext{
${ }^{8}$ The results of Experiment 1 are stronger when the data from the $77 \%$ of participants who responded within $630 \mathrm{~ms}$ of the onset of the prime are examined alone. Because of the $33 \mathrm{~ms}$ interstimulu s interval between the offset of the silhouette primes and the onset of the line drawings in Experiment 1, the comparable subset of participant s had UP RTs $<550 \mathrm{~ms}$. The UP-P differences obtained in this subset of observers in the $83 \mathrm{~ms}$ SOA condition $(-12.56)$ and the $500 \mathrm{~ms}$ SOA condition ( -19.84$)$ remained significantl y greater than zero, $t(14)=3.25, p<.01$, two tailed, and $t(12)=2.98, p<.02$, two tailed.

${ }^{9}$ Uncoupling trials in Experiment 2 afford this analysis because experimental primes were not used in the uncoupling blocks of Experiment 2.
} 
was observed in the priming block, or in any other condition, as can be seen in the bottom panel of Table 2, all $p$ s $>.05$.

\section{DISCUSSION}

In the $83 \mathrm{~ms}$ and the $500 \mathrm{~ms}$ SOA conditions of Experiment 1, and the $50 \mathrm{~ms}$ condition of Experiment 2, observers required more time to accurately classify real line drawings that were preceded by experimental silhouettes than line drawings preceded by control silhouettes. The contours of experimental silhouettes sketched a basic level object along their outer (ground) side that was the same as that portrayed by the line drawing. The increased latency may reflect inhibition of object memories matched in the course of perceptual organization by regions determined to be grounds (see Peterson et al., 2000). Hence, these results are consistent with the proposal that in the course of figure assignment, object memories are accessed for both sides of contours, including the side of the contour later assigned ground status. Given that a good fit to an object memory is possible only when the spatial relationships between the parts has been properly specified, these effects indicate that parts are bound together before ground status is determined. Thus, it is not true that attention to figures is necessary to bind the parts together.

Experiments are currently underway to test whether attention to the spatial location of the prime is necessary to obtain effects such as those presented here. If it is, that would be consistent with recent research showing that attention is required for many perceptual phenomena (e.g., Mack \& Rock, 1998; Nakayama \& Joseph, 1998). Given that the present results reveal aspects of perceptual organization that do not result in conscious awareness, however, they may be observed even when attention is allocated elsewhere (see Moore \& Egeth, 1997). Even if attention to the spatial location of the prime is shown to be necessary for these effects, the present results indicate that neither object files created for figures (but not for grounds) nor attention to those object files is necessary for access to object memories.

The inhibition obtained in the present experiments might be considered similar to negative priming (for review see Neill, Valdes, \& Terry, 1995). Indeed, Treisman and DeSchepper (1996) reported that observers required to match a comparison shape to one of two novel figures on a probe trial were slower when the comparison shape was putatively seen as a ground on a prime trial than when it was a new novel figure. On the basis of these results, Treisman and DeSchepper (1996) proposed that memories for the shapes of grounds are stored even though they are novel, and have not been seen consciously. The question of whether or not object memories are established for novel shapes of grounds is an important one that requires further investigation (e.g., see Lampignano \& Peterson, 2001; Luola, Kourtzi, \& Shiffrar, 2000). However, we were addressing a different question in the present paper. We were 
concerned here with the question of whether or not memories of known objects are accessed in the course of figure assignment. Accordingly, in order not to confuse the two issues, we constructed the experimental primes so that the shape sketched on the high denotative side of their contours (i.e., the outside or the ground side) was not identical to the shape of the subsequently viewed line drawing. Instead, the high denotative side of the silhouette portrayed a different object with the same name as the object portrayed by the line drawing. Any effect of the prime on the line drawing target then must be mediated by a preexisting object memory that can be accessed by different exemplars of the same object (e.g., two different sea horses, or two different owls). Consequently, the latency to respond to the line drawings used in the present experiments can be used to assess whether or not pre-existing object memories have been accessed for ground regions in the course of figure assignment.

Peterson et al. (2000) proposed that, when the cues strongly favour the assignment of figural status on one side of a contour and not the opposite side, processes assessing configural cues and accessing object memories on that opposite side would be inhibited. In the present experiments, this predicted inhibition was observed in the shortest SOA conditions. On the assumption that figure-ground assignment occurs early in the course of perceptual processing, an assumption supported by recent neurophysiological evidence (e.g., Zipser, Lamme, \& Schiller, 1996), it is under such short SOA conditions that inhibition linked to figure and ground determination would be expected. The inhibition we observed was short lived; suggesting it is not the same as negative priming (see Neill et al., 1995). The inhibition observed in the present experiments is attributed to the fact that a region matching the object memory accessed by a line drawing was assigned ground status rather than figure status in experimental primes. In contrast, in negative priming experiments, the ignored elements (to which negative priming accrued) were always presented as figures rather than grounds. We expect that different inhibitory mechanisms account for these two types of effects.

The results of the present experiment are relevant to an alternative interpretation that has been offered for our previous experiments investigating object memory effects on figure assignment. In our previous research, participants made direct reports indicating which of two adjacent regions appeared to be the figure. One of the regions (the high denotative region) portrayed a known mono-oriented object. Participants were more likely to report seeing high denotative regions as figures when the stimuli were presented in an upright orientation rather than an inverted orientation. (In the upright orientation the objects portrayed by high denotative regions were shown in their canonical orientation.) We took these previous results as evidence that object memories can be accessed early in the course of perceptual organization (i.e., while Gestalt configural cues and depth cues are being assessed) and can affect which of two adjacent regions is assigned figural status. We reasoned that effects of object 
memories were not observed for inverted stimuli because object memories are accessed too late in time by inverted stimuli to affect an early perceptual outcome like figure assignment. (See Peterson, 1994a, for a summary.) Driver and Baylis (1995) offered an alternative interpretation of those results resting upon the fact that observers typically recognized the known objects portrayed by the high denotative regions when they see those regions as figures (although recognition need not accompany figural status; see Peterson et al., 2000). Driver and Baylis argued that, once observers recognized some familiar objects in the stimuli, they changed their task to one of searching for known objects rather than reporting the first figure assignment they perceived. In that case, observers' responses might not reflect object memory effects on initial figure assignment. The experiments that are reported in this paper contained no incentives to look for known shapes in the grounds of the prime silhouettes. Hence, they do not lend themselves to the alternative interpretation offered by Driver and Baylis (1995). Instead, these results show that in the course of figure assignment, object memories are accessed (and inhibited) for high denotative regions that are ultimately perceived to be shapeless grounds.

In conclusion, the results of the present experiments, combined with our previous results, show that the parts lying on both sides of contours are bound together sufficiently well to enable access to object memories early in the course of perceptual organization. This binding of parts and their spatial relationships clearly does not depend upon attention to figures and/or to object files created for figures but not for grounds. It remains possible that another form of binding may be needed for conscious perception of definite shape and conscious object recognition, both of which occur for figures but not for grounds (e.g., see Crick \& Koch, 1990). One reason the results obtained in the present experiment support different conclusions than the results of visual search experiments (e.g., Wolfe \& Bennett, 1997) is that the present experiment assesses aspects of perceptual organization that occur outside of conscious awareness, whereas visual search experiments assess conscious perception.

\section{REFERENCES}

Baylis, G.C., Tipper, S.P., \& Houghton, G. (1997). Externally cued and internally generated selection: Differences in distractor analysis and inhibition. Journal of Experimental Psychology: Human Perception and Performance, 23, 1617-1630.

Biederman, I. (1987). Recognition by components: A theory of human image understanding. Psychological Review, 94, 115-147.

Cave, C.B., \& Kosslyn, S.M. (1993). The role of parts and spatial relation s in object identification . Perception, 22, 229-248.

Crick, F., \& Koch, C. (1990). Towards a neurobiological theory of consciousness. Seminars in Neuroscience, 2, 263-275. 
Driver, J., \& Baylis, G.C. (1995). One-sided edge assignment in vision: 2. Part decomposition, shape description, and attention to objects. Current Directions in Psychological Science, 4, 201-206.

Driver, J., \& Baylis, G.C. (1998). Attention and visual object segmentation. In R. Parasuraman (Ed.), The attentive brain (pp. 299-325). Cambridge, MA: The MIT Press.

Hochberg, J. (1971). Perception I: Color and shape. In J.W. Kling \& L.A. Riggs (Eds.), Woodworth and Schlossberg's experimental psychology (3rd ed., pp. 395-474). New York: Hold, Rinehart, \& Winston.

Hummel, J., \& Biederman, I. (1992). Dynamic binding in a neural network for shape recognition . Psychological Review, 99, 480-517.

Kahneman, D., \& Treisman, A. (1984). Changing views of attention and automaticity. In R Parasuraman (Ed.), Varieties of attention (pp. 29-61). New York: Academic Press.

Kroll, J.F., \& Potter, M.C. (1984). Recognizing words, pictures, and concepts: A comparison of lexical, object, and reality decision s. Journal of Verbal Learning and Verbal Behavior, 23, 3966.

Lampignano, D.W., \& Peterson, M.A. (2001, March). Memories for novel shapes seen as grounds? Poster presented at the meeting of the Cognitive Neuroscience Society, New York, USA.

Luola, F., Kourtzi, Z., \& Shiffrar, M. (2000). Surface segmentation cues influence negative priming for novel and familiar shapes. Journal of Experimental Psychology: Learning, Memory, and Cognition, 26, 929-944.

Mack, A., \& Rock, I. (1998). Inattentional blindness. Cambridge, MA: The MIT Press.

Moore, C.M., \& Egeth, H. (1997). Perception without attention: Evidence grouping under conditions of inattention. Journal of Experimental Psychology: Human Perception and Performance, 23, 339-352.

Nakayama, K., \& Joseph, J.S. (1998). Attention, pattern recognition, and pop-out visual search. In R. Parasuraman (Ed.), The attentive brain (pp. 279-298). Cambridge, MA: The MIT Press.

Neill, W.T., Valdes, L.A., \& Terry, K.M. (1995). Selective attention and the inhibitory control of cognition. In F.N. Dempster \& C.J. Brainerd (Eds.), Interference and inhibition in cognition. San Diego: Academic Press.

Peterson, M.A. (1994a). Shape recognition can and does occur before figure-ground organization. Current Directions in Psychological Science, 3, 105-111.

Peterson, M.A. (1994b). The proper placement of uniform connectedness . Psychonomic Bulletin and Review, 1, 509-514.

Peterson, M.A. (1999). On the role of meaning in organization. Intellectica, 28, 37-51.

Peterson, M.A. (2000). Object perception. In E.B. Goldstein (Ed.), Blackwell handbook of perception. Oxford, UK: Blackwell.

Peterson, M.A., de Gelder, B., Rapcsak, S.Z., Gerhardstein, P.C., \& Bachoud-Lévi, A.-C. (2000). Object memory effects on figure assignment: Consciou s object recognitio $\mathrm{n}$ is not necessary or sufficient. Vision Research, 40, 1549-1567.

Peterson, M.A., \& Gibson, B.S. (1993). Shape recognition contribution s to figure-ground organization in three-dimensional display. Cognitive Psychology, 25, 383-429.

Peterson, M.A., \& Gibson, B.S. (1994a). Object recognition contribution s to figure-groun d organization: Operations on outlines and subjectives contours. Perception \& Psychophysics, 56, 551-564.

Peterson, M.A., \& Gibson, B.S. (1994b). Must shape recognition follow figure-ground organization? An assumption in peril. Psychological Science, 5, 253-259.

Peterson, M.A., Harvey, E.H., \& Weidenbacher, H.L. (1991). Shape recognition inputs to figureground organization: Which route counts? Journal of Experimental Psychology: Human Perception and Performance, 17, 1075-1089. 
Rubin, E. (1958). Figure and ground. In D. Beardslee (Ed.), Readings in perception (M. Wertheimer, Trans.; pp. 35-101). Princeton, NJ: Van Nostrand. (Original work published 1915.)

Sejnowski, T.J., \& Hinton, G.E. (1987). Separating figure from ground with a Boltzmann machine. In M. Arbib \& A. Hanson (Eds.), Vision, brain, and cooperative computation. Cambridge, MA: MIT Press.

Snodgrass, J.G., \& Vanderwart, M. (1980). A standardize d set of 260 pictures: Norms for name agreement, image agreement, familiarity, and visual complexity. Journal of Experimental Psychology: Human Learning and Memory, 2, 174-215.

Treisman, A., \& DeSchepper, B. (1996). Object tokens, attention, and visual memory. In T. Inui \& J.L. McClelland (Eds.), Attention and performance XVI: Information integration in perception and communication (pp. 15-46). Cambridge, MA: MIT Press.

Treisman, A., \& Gelade, G. (1980). A feature-integratio n theory of attention. Cognitive Psychology, 12, 97-136.

Vecera, S.P., \& O' Reilly, R.C. (1998). Figure-ground organization and object recognition processes: An interactive account. Journal of Experimental Psychology: Human Perception and Performance, 24, 441-462.

Wolfe, J.M., \& Bennett, S.C. (1997). Preattentive object files: Shapeles s bundles of basic feature s. Vision Research, 37, 25-43.

Zipser, K., Lamme, V.A., \& Schiller, P.H. (1996). Contextual modulation in primary visual cortex. The Journal of Neuroscience, 16, 7376-7389.

\section{APPENDIX}

\section{Set A}

axe

bell

bone

boot

butterfly

coffee pot

dog

eagle

face

grapes

hand

lamp

light bulb

locomotive

palm tree

pig

rhinoceros

spray bottle

umbrella

woman

wrench

\section{Set B}

anchor

duck

elephant

faucet

flower

foot

guitar

house

hydrant

jet plane

leaf

Mickey Mouse

owl

pine tree

pineapple

rabbit

sea horse

snow man

teddy bear

trumpet

watering can 